\title{
Insulin resistance is associated with a modest increase in inflammation in subcutaneous adipose tissue of moderately obese women
}

\author{
T. McLaughlin • A. Deng • O. Gonzales • M. Aillaud • \\ G. Yee • C. Lamendola • F. Abbasi - A. J. Connolly • \\ A. Sherman • S. W. Cushman • G. Reaven • P. S. Tsao
}

Received: 21 May 2008 / Accepted: 11 August 2008 / Published online: 30 September 2008

(C) Springer-Verlag 2008

\begin{abstract}
Aims/hypothesis We have previously described differences in adipose cell size distribution and expression of genes related to adipocyte differentiation in subcutaneous abdominal fat obtained from insulin-sensitive (IS) and -resistant (IR) persons, matched for degree of moderate obesity. To determine whether other biological properties also differ between IR and IS obese individuals, we quantified markers of inflammatory activity in adipose tissue from overweight IR and IS individuals.

Methods Subcutaneous abdominal tissue was obtained from moderately obese women, divided into IR $(n=14)$ and IS $(n=19)$ subgroups by determining their steady-state plasma glucose (SSPG) concentrations during the insulin suppression test. Inflammatory activity was assessed by comparing expression of nine relevant genes and by
\end{abstract}

T. McLaughlin $(\bowtie)$

Department of Medicine, Division of Endocrinology,

Stanford University School of Medicine,

300 Pasteur Drive, Rm S025,

Stanford, CA 94305-5103, USA

e-mail: tmclaugh@stanford.edu

A. Deng $\cdot$ O. Gonzales $\cdot$ M. Aillaud $\cdot$ G. Yee $\cdot$ C. Lamendola $\cdot$

F. Abbasi $\cdot$ G. Reaven $\cdot$ P. S. Tsao

Department of Cardiovascular Medicine,

Stanford University School of Medicine,

Stanford, CA, USA

\section{A. J. Connolly}

Department of Pathology,

Stanford University School of Medicine,

Stanford, CA, USA

A. Sherman - S. W. Cushman

National Institute of Diabetes and Digestive and Kidney Diseases,

National Institutes of Health,

Bethesda, MD, USA immunohistochemical quantification of CD45- and CD68containing cells.

Results SSPG concentrations were approximately threefold higher in IR than in IS individuals. Expression levels of CD68, EMR1, IL8, IL6 and MCP/CCL2 mRNAs were modestly but significantly increased $(p<0.05)$ in IR compared with IS participants. Results of immunohistochemical staining were consistent with gene expression data, demonstrating modest differences between IR and IS individuals. Crown-like structures, in which macrophages surround single adipocytes, were rarely seen in tissue from either subgroup.

Conclusions/interpretation A modest increase in inflammatory activity was seen in subcutaneous adipose tissue from IR compared with equally obese IS individuals. Together with previous evidence of impaired adipose cell differentiation in IR vs equally obese individuals, it appears that at least two biological processes in subcutaneous adipose tissue characterize the insulin-resistant state independent of obesity per se.

Trial registration ClinicalTrials.gov NCT00285844

Funding The study was funded by grants from the National Institutes of Health/National Institute of Diabetes and Digestive and Kidney Diseases (NIDDK) and by the NIDDK Intramural Research Program.

Keywords Adipocyte - Adipose tissue - Inflammation · Insulin resistance $\cdot$ Obesity

$\begin{array}{ll}\text { Abbreviations } \\ \text { CT } & \text { cycle threshold } \\ \text { IR } & \text { insulin-resistant } \\ \text { IS } & \text { insulin-sensitive } \\ \text { IST } & \text { insulin suppression test } \\ \text { MMLV } & \text { Maloney murine leukaemia virus }\end{array}$


MCP1 monocyte chemotactic protein 1

SSPG steady-state plasma glucose

SSPI steady-state plasma insulin

\section{Introduction}

Although it has been known for more than 40 years that overweight/obese individuals are more likely to be insulinresistant $[1,2]$, it is still not clear why this is the case. Our lack of understanding is compounded by the fact that not all obese individuals are insulin-resistant [3, 4], and we have recently published evidence that risk factors for type 2 diabetes and cardiovascular disease were markedly attenuated in the most insulin-sensitive third of 211 apparently healthy, obese individuals $\left(\mathrm{BMI} \geq 30.0<35.0 \mathrm{~kg} / \mathrm{m}^{2}\right.$ ) compared with the most insulin-resistant third [5]. To gain insight into why equally obese individuals can differ so dramatically in insulin sensitivity, we have recently compared [6] adipose cell size distribution and gene expression profiles of abdominal subcutaneous tissue obtained from equally obese individuals, subdivided into insulin-resistant (IR) and insulin-sensitive (IS) subgroups. The results of that study indicated that obese, IR individuals have a relative increase in the proportion of small to large abdominal subcutaneous adipose cells compared with equally obese IS persons. In addition, expression of several genes related to adipose cell differentiation was two- to threefold lower in the IR individuals, suggesting that insulin resistance is characterised by a defect in adipose cell differentiation and fat storage capacity. Such a defect could contribute to insulin resistance by impairing the storage of excess triacylglycerol in adipose tissue, with its diversion to sites such as liver, muscle and pancreas, leading to metabolic abnormalities that characterise insulin resistance and its complications, such as fatty liver and type 2 diabetes.

On the other hand, other differences in the cellular and molecular function of adipose cells and/or tissue may exist that contribute to insulin resistance. In this regard, there is evidence that inflammation may play a role. For example, multiple regression analysis demonstrated that plasma $\mathrm{C}$ reactive protein concentrations were significantly independently related to BMI, systolic blood pressure and insulin sensitivity in a tri-ethnic study [7]. Furthermore, obesity appears to be associated with macrophage accumulation in adipose tissue [8], and studies in Pima Indians have demonstrated increased expression of inflammation-related genes in both subcutaneous tissue [9] and cultured preadipocytes [10] obtained from obese compared with nonobese individuals. However, the latter studies were performed in extremely obese individuals and none of these studies differentiated between the adverse effects of obesity per se from those related to insulin resistance. Since obesity is not synonymous with insulin resistance and a significant portion of moderately obese individuals are insulin-sensitive $[3,4]$, we initiated the present study to see whether insulin resistance is associated with inflammatory activity in adipose tissue independent of obesity. To accomplish this goal we stratified equally obese individuals into IR and IS subgroups and compared markers of inflammatory activity in subcutaneous abdominal adipose tissue.

\section{Participants and methods}

Participants The participants included 33 moderately obese, otherwise healthy women selected from a larger pool recruited to participate in research studies that entailed both quantification of insulin-mediated glucose disposal using a modified insulin suppression test (IST, see below) and a subcutaneous peri-umbilical adipose tissue biopsy. All participants were recruited by newspaper advertisement in the cities surrounding Stanford University. Participants were required to be 35-65 years of age, free of major organ disease, non-diabetic as defined by fasting plasma glucose concentration $<7.0 \mathrm{mmol} / \mathrm{l}$, with stable body weight for 3 months, not engaged in a weight loss programme, and not taking lipid-lowering medications, including fish oil/alpha omega fatty acids, steroid preparations or medications for weight loss. Participants with a history of eating disorder, bariatric surgery or liposuction were excluded, as were pregnant or lactating participants. From the larger participant pool we identified participants with a BMI $\geq$ $27.0<35.0 \mathrm{~kg} / \mathrm{m}^{2}$ and whose IST results put them in the highest or lowest 40th percentile of insulin sensitivity. The study was approved by the Stanford University Human Subjects Committee and all participants gave written, informed consent.

Quantification of insulin-mediated glucose disposal and other clinical measurements Insulin-mediated glucose disposal was quantified by a modification [11] of the IST as originally described and validated [12, 13]. Briefly, participants were infused for $240 \mathrm{~min}$ with octreotide $\left(0.27 \mu \mathrm{g} \mathrm{m}^{-2} \mathrm{~min}^{-1}\right.$ ) (to suppress endogenous insulin secretion), insulin (25 $\mathrm{mU} \mathrm{m}^{-2} \mathrm{~min}^{-1}$ ) and glucose $\left(240 \mathrm{mg} \mathrm{m}^{-2} \mathrm{~min}^{-1}\right)$. Blood was drawn at $10 \mathrm{~min}$ intervals from 210 to $240 \mathrm{~min}$ of the infusion to measure plasma glucose and insulin concentrations, and the mean of these four values was used as the steady-state plasma insulin (SSPI) and glucose (SSPG) concentration for each individual. As SSPI concentrations are similar in all participants during these tests, the SSPG concentration provides a direct measure of the ability of insulin to mediate disposal of an infused glucose load; the higher the SSPG concentration, the more insulin-resistant the individual. Using the distri- 
bution of SSPG concentrations in 449 apparently healthy individuals with BMI distribution similar to that in National Health and Nutrition Examination Surveys (NHANES) [14], we classified those individuals whose SSPG concentrations put them in the upper $40 \%$ of the reference group as being IR and those whose SSPG concentrations were in the lower $40 \%$ as being IS. Participants not fitting into these operational definitions of insulin resistance and sensitivity were excluded from further study.

Other clinical and laboratory measurements After a $12 \mathrm{~h}$ overnight fast, plasma glucose, insulin and lipid/lipoprotein concentrations were measured as previously described $[4,5]$. Other experimental measurements included weight, measured with the participant in light clothing and bare feet; height; BMI, calculated as weight $(\mathrm{kg})$ divided by height $\left(\mathrm{m}^{2}\right)$; waist circumference, measured at endexpiration as the point midway between the iliac crest and lower costal margin; race/ethnicity; and blood pressure (average of six readings taken after sitting quietly for $5 \mathrm{~min})$.

Subcutaneous abdominal fat biopsy and quantitative real time PCR for markers of inflammation Subcutaneous abdominal fat biopsies were performed under sterile conditions by scalpel incision inferior to the umbilicus, as previously described [6]. Total RNA was extracted from flash-frozen biopsy samples using TRIzol (Life Technologies, Gaithersburg, MD, USA) and the Adipose Tissue RNAeasy kit (Qiagen, Valencia, CA, USA) according to the manufacturers' instructions. After DNase treatment, cDNA was synthesised from $5 \mathrm{mg}$ of total RNA using Maloney murine leukaemia virus reverse transcriptase (SuperScript II kit; Invitrogen). Taqman primer/probe sets for markers of inflammatory cell infiltrate $(C D 14, C D 45$ [also known as PTPRC] and CD68), egf-like module containing, mucinlike, hormone receptor-like 1 (EMRl), the interleukin genes IL6 and IL8, inducible nitric oxide synthase (iNOS, also known as $N O S 2 A$ ), monocyte chemotactic protein-1 (CCL2, also known as $M C P 1$ ), tumour necrosis factor ( $T N F$ - $\alpha$, also known as $T N F$ ) and $18 \mathrm{~S}$ ribosomal RNA were obtained from Applied Biosystems (Foster City, CA, USA). Amplification was carried out in triplicate on an ABI Prism 7700 sequence detector at $50^{\circ} \mathrm{C}$ for $2 \mathrm{~min}$ and $95^{\circ} \mathrm{C}$ for $10 \mathrm{~min}$ followed by 40 cycles of $95^{\circ} \mathrm{C}$ for $15 \mathrm{~s}$ and $60^{\circ} \mathrm{C}$ for $1 \mathrm{~min}$ A threshold cycle (CT value) was obtained from each amplification curve and a $\triangle \mathrm{CT}$ value was first calculated by subtracting the $\mathrm{CT}$ value for $18 \mathrm{~S}$ ribosomal RNA from the CT value for each sample. A $\Delta \Delta \mathrm{CT}$ value was then calculated by subtracting the $\Delta \mathrm{CT}$ value of a single insulinsensitive participant (control). Fold changes compared with the control were then determined by raising 2 to the $\Delta \Delta \mathrm{CT}$ power.
Immunohistochemistry Adipose tissue biopsies from a subgroup of 16 participants (seven IS, nine IR) were fixed in formalin and embedded in paraffin. Paraffin sections (5 $\mu \mathrm{m})$ were processed for immunohistochemical analysis using primary antibodies for CD45 and CD68 (Novocastra Laboratories, Newcastle, UK). Immunogenicity was visualised with a standard avidin-biotin-peroxidase technique (Vector Laboratories, Burlingame, CA, USA). All sections were counterstained with Harris haematoxylin. For each section, the proportion of cells expressing these markers was quantified by two blinded observers by light microscopy and normalised per high-power field $(\times 40)$.

Statistical analysis Clinical and laboratory characteristics of the IR and IS groups were compared by Student's unpaired $t$ test. Categorical variables were compared by $\chi^{2}$ analysis. Non-normally distributed variables (triacylglycerol) were log-transformed for analysis. The primary endpoint was the comparison of real-time PCR relative fold expression of inflammatory genes in the IR and IS subgroups by Student's unpaired $t$ test. The secondary endpoint was the between-group comparison of the proportion of cells expressing leucocyte/macrophage markers (CD45 and CD68). The mean number of cells per highpower field was compared between IR and IS by the unpaired Student's $t$ test. Immunohistochemistry was also used in a supporting manner to determine qualitatively whether inflammatory cells were concentrated around single adipose cells (crown-like structures). Statistical significance was accepted at $p<0.05$.

\section{Results}

Demographic, clinical and laboratory characteristics of the IS and IR groups are compared in Table 1. The only statistically significant difference between the two groups was the SSPG concentration, which by design was approximately threefold higher in the IR group than in the IS group.

Figure 1 depicts the relative expression levels of genes associated with inflammation in IS vs IR women. The expression of five of the nine genes compared was significantly greater in adipose tissue from the insulinresistant women (CD68,IL8,IL6, EMR1 and MCP/CCL2), and one difference (CD45) was of borderline significance $(p=0.05)$. It is also apparent from these data that in some cases the magnitude of the differences between the two groups was modest, with increases as low as 5\% (MCP/ CCL2), and in only two cases was the relative activity of the IR individuals increased more than twofold compared with the IS women (IL8 and ILO). 
Table 1 Demographic and clinical characteristics of the two experimental groups

\begin{tabular}{lllc}
\hline Variable & Insulin-sensitive $(n=19)$ & Insulin-resistant $(n=14)$ & $p$ value \\
\hline SSPG (mmol/l) & $4.16 \pm 1.3$ & $12.38 \pm 0.67$ & $<0.0001$ \\
Age (years) & $49 \pm 8$ & $51 \pm 9$ & 0.61 \\
Ethnicity (white, Hispanic, black, Asian) & $17 / 1 / 0 / 1$ & $9 / 2 / 1 / 2$ & 0.33 \\
BMI $\left(\mathrm{kg} / \mathrm{m}^{2}\right)$ & $29.1 \pm 2.1$ & $29.1 \pm 3.2$ & 0.88 \\
Waist circumference (cm) & $97 \pm 10$ & $98 \pm 8$ & 0.90 \\
Systolic BP (mmHg) & $117 \pm 12$ & $125 \pm 15$ & 0.13 \\
Diastolic BP (mmHg) & $68 \pm 6$ & $73 \pm 8$ & 0.08 \\
Fasting glucose (mmol/l) & $5.2 \pm 0.4$ & $4.2 \pm 0.4$ & 0.80 \\
Cholesterol (mmol/l) & $5.15 \pm 0.96$ & $1.23 \pm 0.56$ & 0.27 \\
Triacylglycerol (mmol/l) & $1.05 \pm 0.45$ & $1.34 \pm 0.67$ & 0.40 \\
HDL-cholesterol (mmol/l) & $1.42 \pm 0.47$ & $3.0 \pm 0.65$ \\
LDL-cholesterol (mmol/l) & $3.26 \pm 0.72$ & 0.73 \\
\hline
\end{tabular}

A comparison of the immunohistochemical staining results in a subgroup of IS and IR women is shown in Table 2, and the quantitative analysis of these findings was consistent with the gene expression data in that modest differences between IR and IS individuals were seen. Specifically, cells positive for CD45 (leucocyte marker) and CD68 (macrophage marker), quantified as cells per high-power field, revealed more CD45-positive cells in the IR than in the IS women $(p=0.048)$, and a trend $(p=0.07)$ towards a greater number of CD68-positive cells in the IR group. It can be seen by looking at Tables 1 and 2 that the ages and BMI values of the subgroup of IS and IR individuals whose tissues were analysed were comparable with those of the entire study population.

Figure 2 depicts sections of subcutaneous adipose tissue from IS and IR participants stained for CD45 (leucocyte marker) and CD68 (macrophage marker). Images shown were chosen to be representative of the IS and IR subgroups. Panels a and b represent CD45-stained sections from an IS and an IR participant, respectively, and panels c

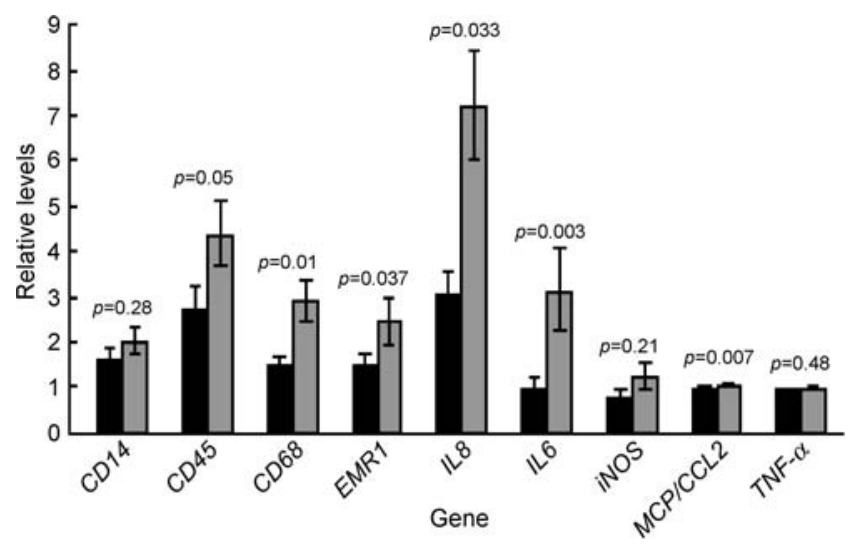

Fig. 1 Inflammatory gene expression $($ mean \pm SE) in adipose tissue from insulin-sensitive (black columns, $n=19$ ) and insulin-resistant (grey columns, $n=14$ ) women. Expression levels are relative to a single insulin-sensitive control (the most insulin-sensitive subject) and d represent CD68-stained sections from an IS and an IR participant, respectively. As with the quantitative data in Table 2, this figure emphasises the small number of CD45positive and CD68-positive cells seen in sections of adipose tissue of either IR or IS participants.

The presence of crown-like structures, in which macrophages surround single adipocytes, was rarely seen in the samples of adipose tissue from either IS or IR participants. Indeed, we found clear evidence of this in only one participant (Fig. 2e), an IS individual (BMI $32.5 \mathrm{~kg} / \mathrm{m}^{2}$ ). Partial crown-like structures were also seen in one IR woman with a BMI of $35.8 \mathrm{~kg} / \mathrm{m}^{2}$ (Fig. 2f), and it is possible that there would be more evidence of these structures if we had studied massively obese women.

\section{Discussion}

The results of this study indicate that the expression of five, possibly six, of the inflammatory genes measured had higher values in adipose tissue of IR women than in that of IS women. In addition, a significantly greater accumulation of CD45-positive cells was observed in adipose tissue of IR individuals. The IR and IS women were equally obese, with values for BMI and waist circumference that were not significantly different, but by selection differed approximately threefold in terms of insulin sensitivity. Thus, we

Table 2 Comparison of inflammatory cells per high-power field (hpf) in the two experimental groups

\begin{tabular}{lccc}
\hline Variable & $\begin{array}{l}\text { Insulin-sensitive } \\
(n=7)\end{array}$ & $\begin{array}{l}\text { Insulin-resistant } \\
(n=9)\end{array}$ & $p$ value \\
\hline Age (years) & $45 \pm 8$ & $46 \pm 9$ & 0.58 \\
BMI (kg/m $\left.{ }^{2}\right)$ & $30.2 \pm 2.1$ & $31.5 \pm 3.5$ & 0.76 \\
CD45 (cells/hpf) & $1.9 \pm 1.5$ & $3.83 \pm 1.85$ & 0.048 \\
CD68 (cells/hpf) & $2.5 \pm 2.5$ & $2.9 \pm 1.7$ & 0.071 \\
\hline
\end{tabular}




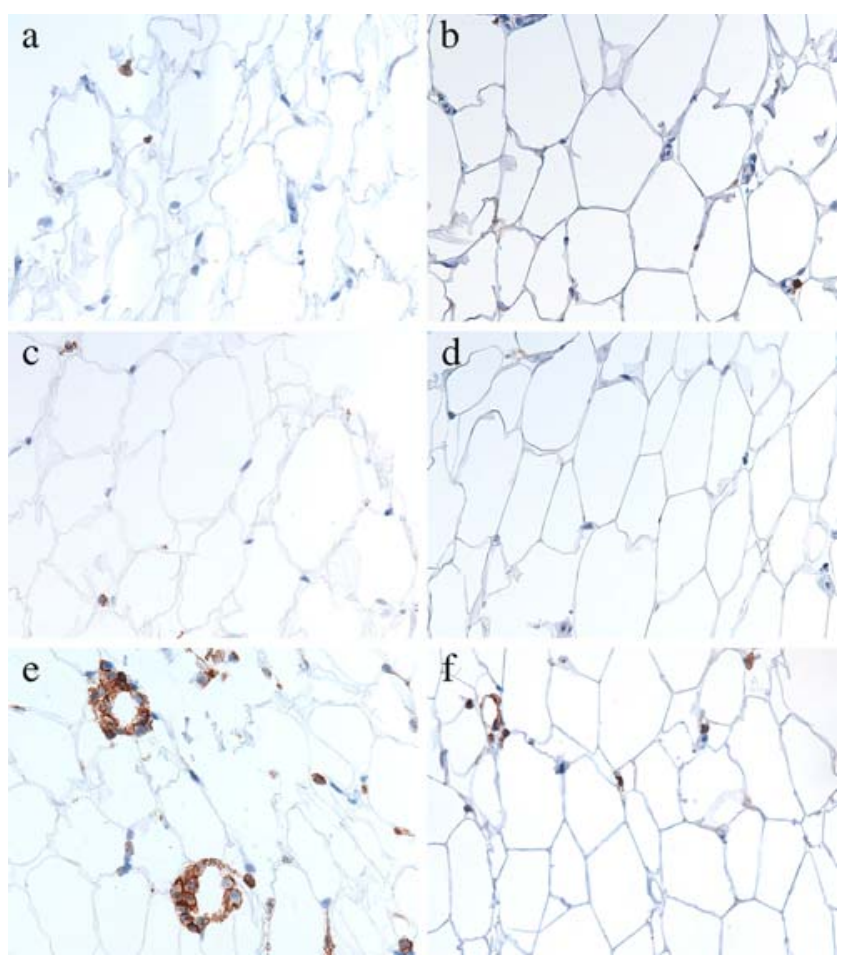

Fig. 2 Representative immunohistochemistry samples from adipose tissue. CD $45^{+}$cells in an insulin-sensitive individual (a) and an insulinresistant individual (b). $\mathrm{CD}^{+} 8^{+}$cells in an insulin-sensitive individual (c) and an insulin-resistant individual (d). CD45 staining for two participants who demonstrated relatively high staining: an IS female with BMI $32.5 \mathrm{~kg} / \mathrm{m}^{2}$ (e) and an IR female with BMI $35.8 \mathrm{~kg} / \mathrm{m}^{2}$ (f)

believe it justified to conclude that the inflammatory behaviour of subcutaneous adipose tissue differs as a function of insulin sensitivity in women with comparable degrees of moderate obesity.

These findings have several possible interpretations, for example, the inflammatory changes could play a major role in the development of insulin resistance. However, the fact that the differences in inflammatory gene expression in the adipose tissue of IR vs IS individuals were modest and that CD45-positive and CD68-positive cells were seen relatively rarely in the adipose tissue, irrespective of the degree of insulin sensitivity, seems to make this possibility less likely. Alternatively, inflammation might be a consequence of insulin resistance, or of adipose cell stress that results from relative inability to store triacylglycerol in existing adipose cells. It seems most reasonable to conclude that although inflammation in adipose tissue may contribute to the development of insulin resistance, it is unlikely to be the major factor in moderately obese individuals. Perhaps the most prudent way to view our results is that they identify another manner in which the adipose tissue of equally obese individuals varies as a function of dramatic differences in insulin sensitivity. In our previous study [6] we described a shift in fat cell distribution, with IR individuals having a relatively greater proportion of smaller fat cells compared with equally obese IS persons. Furthermore, several genetic markers of adipocyte differentiation were significantly decreased in the IR individuals. These findings led to the speculation that fat storage capacity is reduced in obese IR individuals, making it more likely that excess calories will be deposited as ectopic fat and thus contribute to the loss of insulin sensitivity. The current findings suggest that the adipose tissue of equally obese individuals differs in an additional manner, with evidence of modestly increased inflammation in IR relative to IS individuals.

Our findings that inflammatory changes are increased in subcutaneous adipose tissue from IR compared with equally obese IS women must be viewed in light of the fact that comparisons of IR with IS individuals were performed only in women and similar differences may not be observed in men. Since the two groups of women differed in terms of insulin sensitivity, but not in terms of either BMI or waist circumference, the inflammatory changes observed in the adipose tissue of IR women seem likely to be related to insulin resistance rather than adiposity per se. The fact that our study population was only moderately overweight/obese may account for why the inflammatory changes we noted in adipose tissue of IR women were modest compared with several previous studies performed in relatively massively obese rodents and people [8-10, 15-18]. For example, reports in animals and humans have suggested that macrophages may surround adipocytes in an enlarged and/or necrotic crown-like structure, that giant multinucleated cells form to scavenge free lipid droplets, and that enlargement of adipose cells may trigger inflammation in adipose tissue via this mechanism [8, 18]. We did not observe this pattern, and although both leucocytes and macrophages were scattered throughout the tissue in the IR and IS participants, in only one participant was evidence of crown-like structures observed: an IS woman who was not particularly obese and did not have a history of trauma that might have contributed to fat 'necrosis'. However, the BMI in our population ranged from $\geq 27.0$ to $<35.0 \mathrm{~kg} / \mathrm{m}^{2}$, in contrast to BMI values ranging from 19.4 to $60.1 \mathrm{~kg} / \mathrm{m}^{2}$ in the study by Weisberg and associates, in which $7 / 14$ had a BMI $\geq 40 \mathrm{~kg} / \mathrm{m}^{2}$ [8]. Similarly, in the study by Cinti et al. [18] the most striking changes were seen in the subset of individuals with BMI values $>30<45 \mathrm{~kg} / \mathrm{m}^{2}$. Since these earlier studies defined a relationship between the degree of obesity and evidence of inflammation, it is not clear what we would have seen had we enrolled participants with a greater degree of excess adiposity.

In conclusion, the results of our previous study showing that differences in cell size distribution and adipocyte differentiation exist as a function of differences in insulin sensitivity [6] plus the present results demonstrating that inflammation is increased in adipose tissue of IR women 
suggest that these differences, and potentially other as yet unidentified changes in adipose tissue, are related to insulin resistance independent of obesity. The changes noted so far provide possible explanations for the fact that insulin sensitivity can differ dramatically among equally obese individuals. In other words, it is not solely fat mass that determines insulin resistance, but rather the biological properties of the adipose tissue. While the relative importance and directionality of the relationships between impaired adipocyte differentiation, inflammation and insulin resistance are unclear, as are potential intermediary mechanisms such as ectopic fat storage or alterations in circulating cytokines, it does seem worthwhile to continue efforts to increase understanding of the biological basis of insulin resistance in association with obesity.

Acknowledgements Funding for this study was provided by study grants NIH/NIDDK 1 R01 DK071309-01, 5RO1DK071333, 5K23 RR16071 and NIH RR 000070, and by the NIDDK Intramural Research Program.

Duality of interest statement The authors declare that there is no duality of interest associated with this manuscript.

\section{References}

1. Rabinowitz D, Zierler KJ (1962) Forearm metabolism in obesity and its response to intra-arterial insulin. Characterization of insulin resistance and evidence for adaptive hyperinsulinemia. J Clin Invest 41:2173-2181

2. Olefsky JM, Reaven GM, Farquhar JW (1974) Effects of weight reduction on obesity: studies of carbohydrate and lipid metabolism. J Clin Invest 53:64-76

3. Ferrannini E, Natali A, Bell P, Cavallo-Perin P, Lalic N, Mingrone G (1997) Insulin resistance and hypersecretion in obesity. J Clin Invest 100:1166-1173

4. McLaughlin T, Abbasi F, Cheal K, Chu J, Lamendola C, Reaven G (2003) Use of metabolic markers to identify overweight individuals who are insulin resistant. Ann Intern Med 139:802-809

5. McLaughlin T, Abbasi F, Lamendola C, Reaven G (2007) Heterogeneity in the prevalence of risk factors for cardiovascular disease and type 2 diabetes in obese individuals: effect of differences in insulin sensitivity. Arch Intern Med 167:642-648

6. McLaughlin T, Sherman A, Tsao P et al (2007) Enhanced proportion of small adipose cells in insulin-resistant vs insulinsensitive obese individuals implicates impaired adipogenesis. Diabetologia 50:1707-1715

7. Festa A, D, Agostino RH, Howard G, Mykkanan L, Tracy RP, Haffner SM (2000) Chronic subclinical inflammation as part of the insulin resistance syndrome: the Insulin Resistance Atherosclerosis Study (IRAS). Circulation 102:42-47

8. Weisberg SP, McCann D, Desai M, Rosenbaum M, Leibel RL, Ferrante AW Jr (2003) Obesity is associated with macrophage accumulation in adipose tissue. J Clin Invest 112:1796-1808

9. Lee YH, Nair S, Rousseau E et al (2005) Microarray profiling of isolated abdominal subcutaneous adipocytes from obese vs nonobese Pima Indians: increased expression of inflammation-related genes. Diabetologia 48:1776-1783

10. Nair S, Lee YH, Rousseau E et al (2005) Increased expression of inflammation-related genes in cultured preadipocytes/stromal vascular cells from obese compared with non-obese Pima Indians. Diabetologia 48:1784-88

11. Pei D, Jones CNO, Bhargava R, Chen Y-DI, Reaven GM (1994) Evaluation of octreotide to assess insulin-mediated glucose disposal by the insulin suppression test. Diabetologia 37:843-845

12. Shen S-W, Reaven GM, Farquhar JW (1970) Comparison of impedance to insulin mediated glucose uptake in normal and diabetic subjects. J Clin Invest 49:2151-2160

13. Greenfield MS, Doberne L, Kraemer FB, Tobey TA, Reaven GM (1981) Assessment of insulin resistance with the insulin suppression test and the euglycemic clamp. Diabetes 30:387-392

14. Yeni-Komshian H, Carantoni M, Abbasi F, Reaven G (2000) Relationship between several surrogate estimates of insulin resistance and quantification of insulin-mediated glucose disposal in 490 healthy, nondiabetic volunteers. Diabetes Care 23:171-175

15. Hotamisligil GS, Shargill NS, Spiegelman BM (1993) Adipose expression of tumor necrosis factor-alpha: direct role in obesitylinked insulin resistance. Science 259:87-91

16. Uysal KT, Wiesbrock SM, Marino MW, Hotamisligil GS (1997) Protection from obesity-induced insulin resistance in mice lacking TNF-alpha function. Nature 389:610-614

17. Kopp HP, Kopp CW, Festa A et al (2003) Impact of weight loss on inflammatory proteins and their association with the insulin resistance syndrome in morbidly obese patients. Arterioscler Thromb Vasc Biol 23:1042-1047

18. Cinti S, Mitchell G, Barbatelli G et al (2005) Adipocyte death defines macrophage localization and function in adipose tissue of obese mice and humans. J Lipid Res 46:2347-2355 